\title{
Metodología gerencial para facility management ambientalmente sostenible en Bogotá D. C.*
}

[Artículos de investigación]

Jimena Arango Aguirre ${ }^{* * *}$

Recibido: 03 de marzo de 2020

Revisado: 12 de agosto de 2020

Aceptado: 14 de agosto de 2020

Cómo citar este artículo:

Arango Aguirre, J. (2020). Metodología gerencial para facility management ambientalmente sostenible en Bogotá D. C. Signos, Investigación en sistemas de gestión, 13(1). https://doi.org/10.15332/24631140.6343

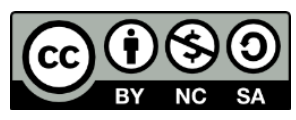

\section{Resumen}

Algunas empresas realizan constantemente soluciones de mantenimiento correctivo y trabajan en un ambiente con malas condiciones locativas y espacios subutilizados que no tienen en cuenta la ergonomía. El presente artículo describe cómo estructurar una metodología gerencial para la administración de proyectos de mantenimientos locativos al aplicar facility management (FM) con un enfoque de sostenibilidad ambiental en edificios corporativos de Bogotá, Colombia. Se realizó un diagnóstico

\footnotetext{
*Artículo de resultado de investigación

** Magíster en Gerencia de Proyectos de la Universidad Militar Nueva Granada. Ingeniera industrial de la Universidad Nacional de Colombia. Bogotá, Colombia. Correo electrónico: u21100141@unimilitar.edu.co, jime2809@gmail.com. ORCID: https://orcid.org/0000-0003-2111-9337. CvLAC: https://scienti.minciencias.gov.co/cvlac/visualizador/generarCurriculoCv.do?cod_rh $=0$ $\underline{001813790}$
} 
de los servicios de gestión de instalaciones más comunes y se analizaron

diferentes enfoques gerenciales y normas ambientales con el fin de aportar sostenibilidad ambiental y uso eficiente a las compañías al conservar sus inmuebles y valorizarlos con el tiempo.

Palabras clave: gestión de instalaciones, contratación externa, sostenibilidad ambiental, mantenimiento, construcción verde.

\section{Management methodology for environmentally sustainable facility management in Bogota D. C.}

\section{Abstract}

Some companies constantly perform corrective maintenance solutions and perform in poor lease conditions environment and underutilized spaces that disregard ergonomics. This article describes how to develop a management methodology for the administration of locative maintenance projects by applying facility management with an environmental sustainability approach in corporate buildings in Bogota, Colombia. A diagnosis of the most common facilities management services was carried out and different management approaches and environmental standards were analyzed in order to provide environmental sustainability and efficient use to companies by preserving their properties and valuing them over time.

Keywords: facilities management, outsourcing, environmental sustainability, maintenance, green building.

\section{Metodologia de gestão para a gestão de instalações (facility management) ambientalmente sustentável em Bogotá D. C.}

\section{Resumo}

Algumas empresas constantemente realizam soluções de manutenção corretiva e trabalham em um ambiente com más condições de locação e 
espaços subutilizados que não levam em conta a ergonomia. Este artigo descreve como estruturar uma metodologia de gestão para o gerenciamento de projetos de manutenção aplicando a gestão de instalações com uma abordagem de sustentabilidade ambiental em prédios corporativos na cidade de Bogotá, na Colômbia. Um diagnóstico dos serviços de gerenciamento de instalações mais comuns foi feito e diferentes abordagens de gerenciamento e padrões ambientais foram analisados a fim de proporcionar sustentabilidade ambiental e uso eficiente às empresas, conservando suas instalações e aumentando seu valor ao longo do tempo.

Palavras-chave: gestão de instalações, terceirização, sustentabilidade ambiental, manutenção, construção verde.

\section{Introducción}

El desarrollo constructivo y la industrialización han generado impactos en la relación edificación-entorno, lo que incrementa los costos ambientales por el excesivo consumo energético y las emisiones contaminantes, y obliga a implementar diseños, mantenimientos y adecuaciones para aprovechar los recursos naturales (Secretaría Distrital de Ambiente, 2019). Históricamente, se han enfrentado dos términos: el desarrollo económico y la protección al medio ambiente, que a primera vista parecen ser opuestos: para lograr uno habría que sacrificar el otro. Sin embargo, existe un enfoque llamado desarrollo sostenible que estudia cómo proceder con el desarrollo económico sin causar daños en el medio ambiente (Chi-man et ál., 2015).

Debido al pensamiento de que "el sistema que importa es la economía y la naturaleza se relega a la función de proveedora de recursos y servicios naturales, y a sumidero de los desechos producidos por la actividad humana" (Gallopín, 2003, p. 13), se infiere que en el afán de producir se ha descuidado la naturaleza. Por lo tanto, de ahora en adelante se debe tener 
una utilización inteligente de los recursos naturales. La sostenibilidad ambiental en las instalaciones físicas hace referencia a la preservación del ambiente por medio de la existencia de relaciones importantes entre la sociedad y la naturaleza.

En el entorno hay influencias económicas, políticas y tecnológicas que afectan la construcción ecológica, por lo que se debe proponer un desarrollo sostenible para el bienestar de las personas en el futuro. Según Gallopín (2003), "es cada vez más evidente que la búsqueda de la sostenibilidad y del desarrollo sostenible exige integrar factores económicos, sociales, culturales, políticos y ecológicos” (p. 7).

$\mathrm{Al}$ aplicar la sostenibilidad ambiental se aprovechan recursos naturales como el agua, el viento, la radiación solar y la vegetación, mediante mantenimientos y adecuaciones que buscan cambiar favorablemente las condiciones ambientales de los usuarios en las instalaciones físicas y mejorar la calidad del aire, la iluminación y el confort térmico (Secretaría Distrital de Ambiente, 2019). Las instalaciones que aplican adecuaciones amigables con el medio ambiente, como la certificación de edificios sostenibles LEED (leadership in energy \& environmental design) obtienen un arrendamiento de mayor valor que instalaciones que no tienen en cuenta estas mejoras. Además, esta práctica sirve como estrategia de marketing para mejorar la imagen corporativa del edificio (Chi-man et ál., 2015). Esta conciencia de las partes interesadas (accionistas, gobierno, cliente, proveedores) por el medio ambiente ha presionado a las empresas a ser más responsables con este tema (Chi-man et ál., 2015).

Para lograr costos eficientes en el sistema de mantenimiento y un funcionamiento eficaz de las instalaciones físicas, es necesaria la planificación estratégica a largo plazo para las diferentes funciones de mantenimiento. La primera decisión estratégica de mantenimiento es la 
categorización de riesgos, en cuanto a los elementos de construcción, de acuerdo a los incidentes presentados y su causa raíz (Yousefli et ál., 2017).

La gestión de instalaciones ofacility management (FM) ejecutada eficazmente podría mejorar el rendimiento de las instalaciones, gracias al control de costos y la mejora de calidad. La efectiva gestión de instalaciones físicas se ve reflejada en su valorización y un servicio de calidad a los usuarios finales, tales como empleados del sector público y privado, inquilinos, etc. (Ogungbile y Oke, 2015).

Con el tiempo, esta disciplina del FM ha ido evolucionando y adquiriendo variadas definiciones (tabla 1). Según Medina y Franco (2014) en los años 70, el término de facility management no era conocido por muchas personas, debido a que este servicio existía por necesidad en las instalaciones físicas para apoyar actividades cotidianas. Su definición se empezó a difundir gracias a un conjunto de facility managers en el año 1978, que reunieron más profesionales del sector para posicionar y reconocer esta disciplina. Esta profesión inició en EE. UU., luego pasó a Inglaterra, Europa, Noruega, Asia, América del Sur y África (Medina y Franco, 2014).

Tabla 1. Definiciones de facility management

\begin{tabular}{|l|l|}
\multicolumn{1}{c|}{ Autor } & \multicolumn{1}{c}{ Definición } \\
\hline Nourse (1990) & $\begin{array}{l}\text { El FM no interfiere en los negocios finales de la empresa en la que se } \\
\text { desarrolla. }\end{array}$ \\
\hline Becker (1990) & $\begin{array}{l}\text { El FM es responsable de coordinar todos los esfuerzos relacionados } \\
\text { con la planificación, el diseño y la gestión de edificios y sus sistemas, } \\
\text { equipos y muebles para mejorar la capacidad de la organización y } \\
\text { competir con éxito en un mundo que cambia rápidamente. }\end{array}$ \\
\hline Bernard (1996) & $\begin{array}{l}\text { El FM son las instalaciones y servicios requeridos para acomodar y } \\
\text { facilitar la actividad comercial. Teniendo esto en cuenta, tienen la } \\
\text { posibilidad de ser totalmente rentables y la gestión de las } \\
\text { instalaciones debe abrazar directamente los tres centros de costo } \\
\text { genérico que incluye: instalaciones, servicios de apoyo y tecnología } \\
\text { de la información. }\end{array}$ \\
\hline
\end{tabular}




\begin{tabular}{|l|l|}
\hline \multicolumn{1}{|c|}{ Autor } & \multicolumn{1}{c|}{ Definición } \\
\hline Alexander (1999) & $\begin{array}{l}\text { El alcance de la disciplina abarca todos los aspectos de la propiedad, } \\
\text { el espacio, el control del medio ambiente, la salud y la seguridad, y } \\
\text { servicios de apoyo. }\end{array}$ \\
\hline $\begin{array}{l}\text { Hinks y McNay } \\
\text { (1999) }\end{array}$ & $\begin{array}{l}\text { El FM se define como la gestión de mantenimiento; las normas de } \\
\text { gestión del espacio y alojamiento; la gestión de proyectos de nueva } \\
\text { construcción y alteraciones; la gestión de las instalaciones generales } \\
\text { de los edificios existentes, y la administración de los servicios de } \\
\text { apoyo asociados. }\end{array}$ \\
\hline Varcoe (2000) & $\begin{array}{l}\text { El FM logra el uso productivo de los espacios de trabajo, lo que } \\
\text { genera una mejora en la gestión y prestación del servicio o producto } \\
\text { core del negocio. }\end{array}$ \\
\hline Nutt (2000) & $\begin{array}{l}\text { La función principal del FM es la gestión de recursos en los niveles de } \\
\text { apoyo estratégico y operativo. Los tipos genéricos de gestión de } \\
\text { recursos centrales de la función de FM son la gestión de recursos } \\
\text { físicos, de recursos humanos, de recursos de información y de } \\
\text { conocimiento. }\end{array}$ \\
\hline (IFMA, 2003) & $\begin{array}{l}\text { La Asociación de Gestión Internacional de Instalaciones define la } \\
\text { administración de las instalaciones como las prácticas de coordinar el } \\
\text { lugar de trabajo físico con las personas y el trabajo de la } \\
\text { organización. }\end{array}$ \\
\hline Shohet y Lavy (2004) & $\begin{array}{l}\text { El FM busca asegurar la mejora continua para el uso de los edificios y } \\
\text { aumentar así el nivel de rendimiento. }\end{array}$ \\
\hline
\end{tabular}

Fuente: elaboración con base en Kurdi et ál. (2011) y Yusefli et ál. (2017).

Para mantener una instalación física se requiere de la ayuda de diferentes áreas como mantenimiento, aseo, seguridad y administración, actividades que siempre se han realizado por separado, pero que en realidad hacen parte del facility management (Díaz y Torres, 2017) y cuya adecuada ejecución trae múltiples beneficios.

Según Sridarran y Gayani (2016), algunos de los beneficios del FM como outsourcing son:

- Costo bajo gracias a la adquisición de elementos en cantidad con aliados estratégicos, lo que contribuye a buenas prácticas en el mercado.

- Mayor atención en el core del negocio, mientras el outsourcing del FM se encarga de las actividades externas con especialistas en este campo 
- Mejor negociación con proveedores especializados

- $\quad$ Riesgos de los servicios prestados en outsourcing asumidos por la empresa encargada.

- Evitar inversiones grandes en la creación de un departamento de FM

- Mayor flexibilidad en el ajuste de cantidad, lugar y calidad de acuerdo con la necesidad del cliente

El FM se debe desarrollar en Colombia para mejorar la competitividad de las empresas y evitar pérdidas de tiempo y dinero al intentar solucionar temas de los cuales no se tiene conocimiento. Esta metodología implementa ahorros que benefician al medio ambiente y a las personas, pues disminuye el consumo eléctrico e hidráulico al utilizar elementos amigables con el medio ambiente y que generan ahorros significativos en el consumo de los recursos.

$\mathrm{Al}$ realizar adecuaciones amigables con el medio ambiente se contribuye a la reducción de concentraciones contaminantes para los ocupantes de oficinas, implementando, por ejemplo, un plan de gestión de residuos. Si se realiza una intervención eficaz, se puede llegar a reducir la huella de carbono, disminuir el consumo de energía y mejorar la satisfacción de los ocupantes, lo que significa que no solo se tiene en cuenta la cantidad, sino también la calidad del espacio.

Si se implementa el FM ambientalmente sostenible en los edificios corporativos, se mejora la calidad de los espacios interiores de las empresas y se crea un mejor ambiente de trabajo para los empleados, con plantas abiertas para una mejor vista a exteriores y luz natural. Es decir, se optimizan cualidades ambientales interiores como el confort térmico, la iluminación, la acústica, etc, y se adecúan las condiciones de los ocupantes en cuanto a climatización, comodidad, comunicación, relaciones interpersonales, comportamiento, desempeño laboral y productividad. 
En el presente artículo se da a conocer el proceso mediante el cual se logra la definición de la metodología gerencial para implementar el FM ambientalmente sostenible en Bogotá mediante el cumplimiento de los diferentes objetivos:

- Diagnosticar los elementos y servicios que contribuyen al mantenimiento locativo en los edificios corporativos en Bogotá.

- Determinar las metodologías gerenciales adaptables al FM.

- Proponer una metodología gerencial que se articule con el FM en la sostenibilidad ambiental de edificios corporativos.

- Validar la metodología en un edificio corporativo que tenga compromiso de sostenibilidad ambiental.

\section{Metodología}

La investigación tuvo un enfoque cualitativo orientado a "comprender los fenómenos, explorándolos desde la perspectiva de los participantes en su ambiente natural y en relación con el contexto" (Hernández-Sampieri y Mendoza, 2018, p. 358), para identificar los servicios más necesarios, la frecuencia y la forma en que se realizan, y conocer las percepciones de clientes, usuarios y personas involucradas en los procesos que suministran información relevante para alcanzar los objetivos propuestos.

En la investigación exploratoria (tabla 2) se indaga sobre fenómenos de los cuales no se tiene toda la información necesaria. Por medio de esta investigación se identifican conceptos y escenarios más profundos que permiten innovar en el tema seleccionado. En la investigación explicativa se entienden las causas de los eventos estableciendo relaciones entre diferentes elementos, hechos o fenómenos (Hernández-Sampieri y Mendoza, 2018). Las técnicas utilizadas fueron observación y entrevista, que permiten evidenciar las opiniones y perspectivas de las personas con 
respecto a su ambiente de trabajo, productividad e impacto hacia el medio ambiente.

Tabla 2. Procedimiento de metodología

\begin{tabular}{|c|c|c|c|}
\hline Objetivo general & Objetivos específicos & Actividades & Resultados \\
\hline \multirow{4}{*}{$\begin{array}{l}\text { Estructurar una } \\
\text { metodología } \\
\text { gerencial para la } \\
\text { administración } \\
\text { de proyectos de } \\
\text { mantenimientos } \\
\text { locativos } \\
\text { aplicando la } \\
\text { gestión del } \\
\text { facility } \\
\text { management en } \\
\text { edificios } \\
\text { corporativos con } \\
\text { un enfoque de } \\
\text { sostenibilidad } \\
\text { ambiental. }\end{array}$} & $\begin{array}{l}\text { Diagnosticar los elementos } \\
\text { y servicios que } \\
\text { contribuyen al } \\
\text { mantenimiento locativo en } \\
\text { los edificios corporativos } \\
\text { en Bogotá. }\end{array}$ & $\begin{array}{l}\text { Se revisa el estado } \\
\text { actual de una muestra } \\
\text { de edificios } \\
\text { corporativos y se } \\
\text { verifican los servicios } \\
\text { implementados y } \\
\text { necesarios para su } \\
\text { mantenimiento. }\end{array}$ & $\begin{array}{l}\text { Diagnóstico } \\
\text { inicial } \\
\text { (observación, } \\
\text { entrevista) }\end{array}$ \\
\hline & $\begin{array}{l}\text { Determinar las } \\
\text { metodologías gerenciales } \\
\text { adaptables al facility } \\
\text { management. }\end{array}$ & $\begin{array}{l}\text { Realizar una revisión } \\
\text { literaria sobre las } \\
\text { metodologías aplicadas } \\
\text { en esta disciplina. }\end{array}$ & $\begin{array}{l}\text { Listado de } \\
\text { metodologías a } \\
\text { implementar }\end{array}$ \\
\hline & $\begin{array}{l}\text { Proponer una metodología } \\
\text { gerencial que se articule } \\
\text { con el facility } \\
\text { management con enfoque } \\
\text { en la sostenibilidad } \\
\text { ambiental en edificios } \\
\text { corporativos. }\end{array}$ & $\begin{array}{l}\text { Encontrar una } \\
\text { metodología desde la } \\
\text { operación, el } \\
\text { mantenimiento y las } \\
\text { alternativas "pasivas". } \\
\text { Solucionar y volver } \\
\text { sostenible el edificio. }\end{array}$ & $\begin{array}{l}\text { Metodología a } \\
\text { implementar }\end{array}$ \\
\hline & $\begin{array}{l}\text { Validar la metodología en } \\
\text { edificio corporativo que } \\
\text { cuente con compromiso de } \\
\text { sostenibilidad ambiental. }\end{array}$ & $\begin{array}{l}\text { Aplicar la metodología } \\
\text { en edificio corporativo. }\end{array}$ & $\begin{array}{l}\text { Aplicación de } \\
\text { metodología y } \\
\text { conclusiones }\end{array}$ \\
\hline
\end{tabular}

Fuente: elaboración propia.

El diseño es documental por ser investigación de tipo cualitativa. Se utiliza la teoría fundamentada que, según Hernández-Sampieri y Mendoza (2018), brinda información de las categorías del proceso y sus vínculos, y proporciona una "teoría que explica un fenómeno o responde al planteamiento”. De acuerdo con estos autores, el análisis se centra en organizar los datos recibidos de las observaciones y entrevistas a los participantes; estos datos se encuentran de diferentes maneras: visuales, auditivos, textos escritos, expresiones verbales y gestos.

Con el análisis cualitativo se busca explorar los datos, imponerles una estructura, describir las experiencias de los participantes, identificar 
posibles patrones, categorías o conceptos para darle un sentido a los datos; entender el contexto de la información; reconstruir hechos e historias; vincular los resultados con el conocimiento disponible, y generar una teoría fundamentada en los datos. En resumen, los pasos para realizar el análisis fueron: recolección, revisión, organización y preparación de datos para el análisis, definición de los criterios y comentarios relevantes.

Según Hernández-Sampieri y Mendoza (2018), la muestra se define en la inmersión sin importar su tamaño, lo importante es entender bien el fenómeno para dar claridad a los cuestionamientos de la investigación. En la investigación cualitativa se van dando diferentes tipos de unidades así: "comenzar con la identificación de ambientes propicios, luego de grupos y, finalmente, de individuos u otro tipo de casos" (Hernández-Sampieri y Mendoza, 2018, p. 435).

\section{Resultados y discusión}

Para lograr un diagnóstico inicial sobre el FM en los edificios corporativos de Bogotá e identificar los servicios y elementos más representativos, se realizó una entrevista con las siguientes preguntas a las personas encargadas de administrar cinco de los edificios corporativos más representativos de Bogotá:

1. Antigüedad de la instalación física

2. Tipo de ocupantes en el edificio

3. ¿Usted cómo definiría el facility management o gestión de instalaciones?

4. ¿De qué manera hacen el mantenimiento en sus instalaciones? ¿Correctivo, preventivo o predictivo?

5. ¿Ha tenido accidentes por culpa del mal estado de las instalaciones físicas? 
6. ¿Tiene personas expertas dedicadas exclusivamente al mantenimiento locativo? Si su respuesta es sí, ¿estas personas son externas o internas?

7. ¿Sus empleados se quejan del confort en su ambiente de trabajo?

8. Para usted, ¿cuáles son las barreras para implementar el facility management?

9. ¿Qué tecnologías amigables con el medio ambiente han implementado y cuáles piensa implementar a futuro?

10. ¿Cuenta con algún tipo de certificación ISO o ambiental? ¿Qué certificación ambiental quisiera alcanzar?

11. ¿Qué impacto tienen las instalaciones ecoeficientes?

12. ¿Ha identificado algún ahorro gracias a las prácticas de facility management ambientalmente sostenible?

13. ¿Los sobrecostos al implementar esta modalidad se ven recuperados? ¿En cuánto tiempo?

La información recopilada corresponde a los siguientes edificios: Centro Empresarial BOG Américas, Edificio World Trade Center Bogotá (WTCB), Edificio Paralelo 108, El Cubo Colsubsidio y Torre Mansarovar. De estos, los primeros tres tienen un área superior a $60000 \mathrm{~m}^{2}$, lo que indica que su operación es más compleja porque tienen mayor cantidad de áreas a mantener en óptimo estado, más personal a cargo, mayor ocupación, más equipos y materiales.

Existen algunas barreras para la correcta gestión e implementación de este tipo de tecnologías, como el tema presupuestal, por no contar con la aprobación del consejo de administración y recaudo de cuota extraordinaria, seguido de no realizar una correcta planeación de mantenimientos y mejoramientos y la falta de cultura ambiental por parte de los dueños, proveedores y usuarios de las instalaciones físicas. La ejecución de mejoras no solo ambientales sino también de mantenimiento general demanda una buena planeación, costos y esfuerzo conjunto. 
Es cierto que se incurre en sobrecostos al momento de implementar tecnologías y materiales amigables con el medio ambiente, pero también se ve recuperado y con ganancias en el tiempo. Por ejemplo, la implementación de luminarias LED ahorra un 20 \% de energía con un retorno de la inversión en 18 meses y además brinda ganancias en los siguientes años ${ }^{1}$.

La mayoría de las personas de los edificios entrevistadas contaban con alguna certificación ambiental: el Centro Empresarial BOG Américas cuenta con la certificación LEED Silver, el WTCB con certificación de baja emisión de huella de carbono, El Cubo Colsubsidio con certificación LEED Oro, y el Edificio Paralelo 108 y la Torre Mansarovar realizan gestiones internas para el cumplimiento ambiental.

El edificio El Cubo es certificado LEED Categoría Oro y los desarrollos amigables con el medio ambiente implementados según el ingeniero de operaciones fueron:

- $\quad$ Consumo de luz natural del $90 \%$ : todos los escenarios utilizan luz natural (ventanas amplias).

- $\quad$ Sistemas de ventilación bioclimática: no se utilizan ventiladores ni motores eléctricos, por lo tanto, no se consume energía.

- $\quad$ Sistemas de agua ahorradores.

- Sistemas de aguas reciclada: se recupera agua de las duchas, lavamanos, aguas lluvias. Se utilizan aguas del subsuelo para lavado de la piscina. Se lleva a un sistema de aguas grises para los sanitarios y orinales, lo que genera un ahorro del $40 \%$ en agua.

- Programa de reciclaje y comunicación a usuarios: cómo manejar desechos y generar conciencia.

\footnotetext{
${ }^{1}$ De acuerdo con la entrevista realizada al administrador y representante legal del edificio World Trade Center Bogotá.
} 
- Disposición de residuos certificada.

- $\quad$ Sistema en el que se crean bacterias aerobias y anaerobias que ayudan a limpiar el agua y sirven para el uso en sanitarios.

- Instalaciones construidas con materiales sostenibles.

- El diseño de fachada ventilada no deja afectar la temperatura.

- Sistema que va graduando iluminación.

- $\quad$ Se tienen jardines en cubierta con plantas en vía de extinción.

- En la cubierta se recupera el agua lluvia.

- Pisos negros plásticos en material reciclable.

El WTCB cuenta con certificación en bajas emisiones de huella de carbono. Las tecnologías implementadas según el administrador fueron:

- $\quad$ Paneles solares de $5.8 \mathrm{kV}$

- Cambio en la tecnología de iluminación con sistemas LED y sensores de movimiento

- $\quad$ Actualización de los equipos de transporte vertical (ascensores) con consumos eficientes en energía

- Actualización de tableros eléctricos de bombas adicionando variadores de velocidad que permiten una mayor eficiencia en consumo energético

- Programa de reciclaje y aprovechamiento de residuos sólidos

- Incremento de áreas de cobertura vegetal

- Uso de transporte alternativo como la bicicleta para los usuarios del edificio

- Actualización de equipos de aire acondicionado, cambiando el refrigerante utilizado por uno más amigable con el medio ambiente

- Actualización de los sistemas de control de plantas eléctricas para tener mayor control sobre los tiempos de encendido, lo que disminuye la emisión de gases de efecto invernadero 
El Centro Empresarial BOG Américas hace uso de los siguientes

desarrollos ambientales y cuenta con la certificación LEED Silver así:

- Centro de acopio para recolección de basuras

- Ventilación mecánica, ahorro de energía (iluminación LED) y sensores de movimiento

- $\quad$ Detectores de humo y red contra incendio

- Reutilización de aguas residuales (una parte de aguas lluvias y otra de lavamanos) para riego de plantas

- Jardines con plantas naturales en el perimetral y terrazas de las torres

Los ahorros identificados gracias a estas prácticas ambientales son principalmente en agua y energía; del $20 \%$ en el edificio WTCB en energía y del $40 \%$ en agua en El Cubo de Colsubsidio. En el edificio Paralelo 108, la administradora indicó que se identificó ahorro en el consumo del agua y en el pago de la factura energía, ya que en este año se han ahorrado 150 millones en energía y 150 millones en agua, y la asistente administrativa de Torre Mansarovar también mencionó que los costos de la energía eran de 18 millones, pero la debido a la implementación de tecnología LED se pagan 12 millones mensuales, lo que genera ahorros significativos.

En general, las personas entrevistadas indicaron que se evidenció un retorno de la inversión gracias a los desarrollos ambientales y confirmaron el impacto positivo que trajo la implementación de estos mantenimientos amigables con el medio ambiente. Esto contribuye a su protección y conservación, influye en los usuarios y valoriza los activos fijos al hacerlos más llamativos.

Según lo anterior, se puede identificar que para lograr cualquier tipo de certificación o desarrollo ambiental en los edificios corporativos es 
necesaria la intervención de un mantenimiento locativo programado, organizado y eficiente.

De acuerdo con el diagnóstico realizado previamente se determinaron los criterios necesarios para el buen funcionamiento del FM ambientalmente sostenible en edificios corporativos (tabla 3) y se determinó la aplicación de cada uno de estos criterios en tres diferentes metodologías: PMBOK (Project Management Body of Knowledge) y Prince2 (Projects in Controlled Environments), que se centran en el manejo de proyectos, y la Norma ISO 14001:2015, que proporciona las bases para el manejo ambiental.

La metodología se entiende como la organización de un grupo de actividades o tareas de manera formal y es una herramienta importante en la gestión de proyectos debido a que establece controles y mide su progreso (Matos y Lopes, 2013).

Tabla 3. Criterios de facility management vs. metodologías

\begin{tabular}{|l|c|c|c|}
\hline \multicolumn{1}{|c|}{ Criterios FM } & \multicolumn{3}{c|}{ Metodologías } \\
\cline { 2 - 4 } & PMBOK & Prince2 & $\begin{array}{c}\text { ISO 14001 } \\
\text { (ambiental) }\end{array}$ \\
\hline Planeación & $\checkmark$ & $\checkmark$ & $\checkmark$ \\
\hline Ejecución & $\checkmark$ & $\checkmark$ & $\checkmark$ \\
\hline Seguimiento & $\checkmark$ & $\checkmark$ & $\checkmark$ \\
\hline Comunicación & $\checkmark$ & $\checkmark$ & $\checkmark$ \\
\hline Recursos & $\checkmark$ & $\checkmark$ & $\checkmark$ \\
\hline Interesados & $\checkmark$ & $X$ & $\checkmark$ \\
\hline Costos & $\checkmark$ & $\checkmark$ & $X$ \\
\hline Calidad & $\checkmark$ & $\checkmark$ & $X$ \\
\hline Riesgos y oportunidades & $\checkmark$ & $\checkmark$ & $\checkmark$ \\
\hline Tiempo & $\checkmark$ & $\checkmark$ & $x$ \\
\hline Ambiental & $X$ & $X$ & $\checkmark$ \\
\hline Entregables & $\checkmark$ & $\checkmark$ & $\checkmark$ \\
\hline
\end{tabular}




\begin{tabular}{|l|c|c|c|}
\multicolumn{1}{c|}{ Criterios FM } & \multicolumn{3}{c|}{ Metodologías } \\
\cline { 2 - 4 } & PMBOK & Prince2 & $\begin{array}{c}\text { ISO 14001 } \\
\text { (ambiental) }\end{array}$ \\
\hline Alcance & $\checkmark$ & $\checkmark$ & $\checkmark$ \\
\hline Mejora continua & $\checkmark$ & $\checkmark$ & $\checkmark$ \\
\hline Compras & $\checkmark$ & $X$ & $\checkmark$ \\
\hline
\end{tabular}

Fuente: elaboración propia.

De acuerdo con la tabla 3, Prince2 no cumple con todos los criterios necesarios para implementar el FM a profundidad. La guía PMBOK cuenta con diez áreas del conocimiento (PMI, 2017), las cuales se ejecutan de acuerdo con la fase del proyecto en la que se encuentren, mientras que en la norma ISO 14001 se maneja el ciclo PHVA (planear, hacer, verificar, actuar) y en cada una de estas fases se aplican componentes similares a las áreas del conocimiento del PMBOK pero desde el ámbito ambiental. El conocimiento de la guía $\mathrm{PMBOK}$ ha avanzado gracias a los profesionales en esta área, quienes con sus buenas prácticas reconocidas en la gestión de proyectos han aportado a su desarrollo (Varajão et ál., 2017).

Según la comparación, se realizó una metodología híbrida teniendo en cuenta el ciclo PHVA del Sistema de Gestión Ambiental ISO 14001:2015 y las áreas de conocimiento de la guía PMBOK, que se complementaron con aspectos ambientales de manera transversal (figura 1). 
Figura 1. Metodología gerencial para la articulación entre el FM y la sostenibilidad ambiental aplicable a edificios corporativos

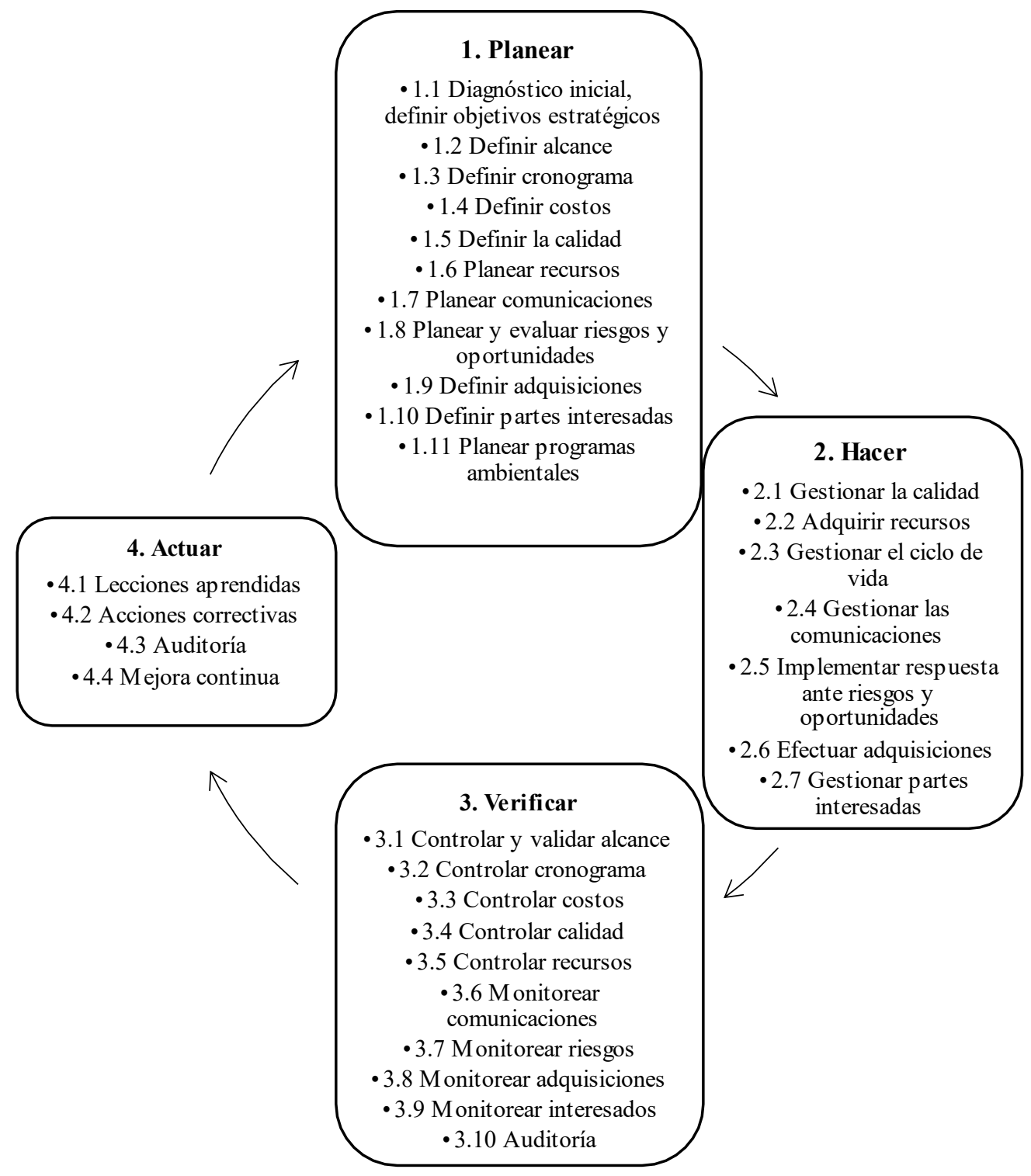

Fuente: elaboración propia.

\section{Planear}

Dentro de esta fase se encuentran todos los procesos que permiten definir un alcance y una línea base a seguir para cumplir los requisitos del cliente y los objetivos planteados. 
Hacer

En esta fase se realiza el trabajo establecido en la planeación. Se ejecuta cada grupo de procesos definidos en el PMBOK y la ISO 14001 de manera conjunta y se engranan sus actividades para cumplir los objetivos planteados.

\section{Verificar}

Corresponde al grupo de actividades y procesos encargados de realizar un seguimiento, análisis, monitoreo y control del proyecto, para evaluar su desempeño e identificar a tiempo posibles cambios y nuevos riesgos que deberán ser validados.

\section{Actuar}

Teniendo en cuenta lo aplicado anteriormente, en esta fase se aplican acciones correctivas y de mejora con el fin de optimizar los procesos, las actividades, el servicio y el sistema.

\section{Validación}

Todo el diseño de la metodología expuesto previamente fue aplicado en el edificio corporativo WTCB (figura 1). Se realizaron registros, documentos y matrices que permitieron una buena, planeación, ejecución, seguimiento y mejora continua del proyecto, para alcanzar finalmente la certificación en la norma ISO 14001:2015, lo que comprueba la validación de dicha metodología. Esta metodología puede ser aplicada en cualquier edificio corporativo. A continuación, se especifican los componentes de la metodología y las actividades más relevantes. 


\section{Planear}

- El primer paso para identificar el contexto interno y externo de la organización fue hacer un diagnóstico mediante la herramienta de análisis DOFA (debilidades, oportunidades, fortalezas y amenazas).

- Después de identificar los aspectos relevantes de la organización con la junta directiva, se definió el alcance del proyecto, se relacionaron las áreas y procesos a incluir en este, y se definieron los entregables desglosados y definidos en la estructura de desglose de trabajo (EDT).

- Después de aprobarse el alcance y los entregables, se elaboró un cronograma para la implementación de la metodología fijando actividades y tiempos de cumplimiento.

- Se verificaron los costos asociados a esta implementación, relacionando el presupuesto del año 2019, que incluyó asesorías, costos de papelería, cafetería, equipos de cómputo, calibración de equipos, etc.

- Se identificó toda la normatividad legal ambiental aplicable, la cual deberá ser actualizada anualmente de acuerdo a nuevas resoluciones $\mathrm{y}$ decretos a los que se les deba dar cumplimiento.

- Se realizó el organigrama para identificar el orden y la jerarquía de cargos identificando quién es el jefe directo y a quién se le debe reportar las tareas realizadas o incidencias, y el perfil de cargo para conocer la formación que deben tener, sus funciones y sus responsabilidades ambientales.

- Para definir canales claros de comunicación, se creó una matriz en donde se especificó qué, quién, a quién, por qué medio y con qué frecuencia se comunica, además de especificar el registro donde se encuentra almacenada la información. 
- Se creó una matriz junto con el responsable de cada área, en donde se identificaron todas las actividades del servicio y sus posibles eventos, efectos e impacto al medio ambiente con una calificación para cada aspecto. Dado que el edificio debe estar preparado para cualquier tipo de emergencia, se creó un plan de respuesta ante emergencias ambientales.

- $\quad$ Las compras y contrataciones fueron planificadas a lo largo del año en un cronograma de mantenimientos especializados. Sin embargo, algunas adquisiciones se realizaron según la necesidad del servicio mediante orden de compra.

- $\quad$ Con el fin de identificar a las personas y entidades afectadas por el proyecto, se creó una matriz en donde se especifican las partes interesadas internas y externas, así como sus necesidades, expectativas, requisitos y frecuencia de seguimiento.

- Se programaron capacitaciones con temas ambientales y se realizó seguimiento al consumo de agua y energía periódicamente.

\section{Hacer}

En esta fase se ejecutó lo consignado en la fase anterior y se realizaron algunos registros propios de la ejecución del proyecto.

- Se realizó el ciclo de vida de cada actividad, con el fin de identificar y controlar el uso de elementos y sustancias que podían hacer daño al medio ambiente.

- A los riesgos y oportunidades calificados con un puntaje mayor a 12, se les realizó un plan de acción en donde se describen las actividades a realizar para mitigar el riesgo o explotar la oportunidad.

- Para formalizar la solicitud de un producto o servicio, se realizaron órdenes de compra. 
- $\quad$ Por seguridad ambiental y buen manejo de los productos a utilizar, a los proveedores y contratistas se les solicitó el envío de las hojas de seguridad de los materiales y productos a manejar en los trabajos.

- $\quad$ En el transcurso del proyecto, se gestionaron las partes interesadas de acuerdo a la matriz de interés-poder, en la que, según su ponderación, se determinó la forma de gestionarlas.

\section{Verificar}

En esta fase se realizó seguimiento y control periódico de lo realizado en la fase anterior.

- $\quad$ Se realizó un seguimiento al cronograma identificando el cumplimiento de las actividades en las fechas programadas.

- Los equipos necesarios para la prestación del servicio se calibraron o verificaron de acuerdo a lo planeado para asegurar una correcta prestación del servicio.

- Se realizaron encuestas anuales para verificar la satisfacción del cliente con el servicio. Se evidenció un aumento en la calificación gracias a la metodología que se ha ido aplicando.

- Los jefes directos realizaron evaluaciones de desempeño a sus trabajadores semestralmente, para calificar su desempeño general, habilidades generales y habilidades propias del cargo, y generar incentivos y formación de acuerdo al resultado obtenido.

- Se le hizo seguimiento a los contratistas y proveedores; de acuerdo a las fallas registradas se les dio una calificación.

- Después de implementar los procedimientos y formatos requeridos, se programaron auditorías periódicas para hacer seguimiento al cumplimiento de los lineamientos establecidos. 


\section{Actuar}

En esta fase se realizaron las correcciones y mejoras al proyecto para generar un ciclo de mejora continua en donde todas las actividades y tareas se pudieran desarrollar optimizando los procesos.

- De acuerdo con las auditorías o no conformidades en el proceso, se debe realizar una acción correctiva.

- Las auditorías se deben realizar periódicamente. En estas se revisa el cumplimiento y la conformidad del sistema de gestión y se emite un informe con los resultados y hallazgos de la visita.

Aplicando todos estos pasos y procedimientos, finalmente la empresa administradora del WTCB alcanzó la certificación en la norma ISO 14001:2015.

\section{Conclusiones}

Como resultado del diagnóstico se obtuvieron datos relevantes para la investigación tales como la planificación de tiempos o cronogramas, la ejecución del presupuesto, el manejo de recursos y proveedores, la definición de los canales de comunicación, las tecnologías amigables con el medio ambiente, los ahorros significativos, entre otros. Después de analizar cinco proyectos diferentes, se encontró que la metodología está en proceso de crecimiento debido a que no se ha formalizado el término de facility management ni su integración con las normas ambientales.

Gracias a la revisión literaria, las entrevistas y la comparación de diferentes metodologías de proyectos y medio ambiente vs. criterios necesarios para el FM, se eligió la combinación de dos metodologías que se ajustan a las necesidades del problema planteado. Se realizó una metodología híbrida para su aplicación en los proyectos de mantenimiento locativo con enfoque ambiental mediante la guía del PMBOK y la norma 
ISO 14001:2015, aplicando de manera conjunta cada uno de sus numerales en cada fase del ciclo PHVA.

La validación de la metodología en el edificio WTCB ha tenido resultados satisfactorios, pues se han organizado los procesos y los usuarios están más conformes con el servicio debido a que encuentran las instalaciones en buenas condiciones y con un impacto bajo al medio ambiente. Esto se ve reflejado en el aumento de ocupación y valorización del edificio a pesar de sus 35 años de antigüedad.

\section{Referencias}

Chi-man, E., Wing-Fai, E. y Yu, K. (2015). The effect of LEED certification on Shanghai's prime office rental value. Journal of Facilities Management, 13(3), 297-310. https://doi.org/10.1108/JFM-10-2014-0033

Díaz, N. y Torres, F. (2017). Servicio integral de mantenimiento locativo orientado a propiedad horizontal [Tesis de especialización]. Universidad Nacional Abierta y a Distancia. http://hdl.handle.net/10596/12435

Gallopín, G. (2003). Sostenibilidad y desarrollo sostenible: un enfoque sistémico. División de desarrollo sostenible y asentamientos humanos. Comisión Económica para América Latina y el Caribe. https://repositorio.cepal.org/handle/11362/5763

Hernández-Sampieri, E. y Mendoza, C. (2018). Metodología de la investigación. Las rutas cuantitativa, cualitativa y mixta. En Metodología de la investigación (pp. 424-559). http://www.ebooks7-24.com.ezproxy.umng.edu.co/?il=6443

Kurdi, M. K., Abdul-Tharim, A. H., Jaffar, N., Azli, M. S., Shuib, M. N. y Ab-Wahid, A. M. (2011). Outsourcing in facilities management - A literature review. Procedia Engineering, 2O, 445-457. https://doi.org/10.1016/j.proeng.2011.11.187

Matos, S. y Lopes, E. (2013). Prince2 or PMBOK - a question of choice. Procedia Technology, 9, 787-794. https://doi.org/10.1016/j.protcy.2013.12.087

Medina, J. y Franco, R. (2014). Análisis de factibilidad de expandir el servicio de la empresa serviactiva de facility management en Guatemala y República Dominicana [Tesis de especialización]. Universidad de la Sabana.

http://hdl.handle.net/10818/12844

SIGNOS, Investigación en Sistemas de Gestión

ISSN: 2145-1389 | e-ISSN: 2463-1140 | DOI: https://doi.org/10.15332/24631140

Vol. 13 N. ${ }^{\circ} 1$ | enero-junio de 2021 
Ogungbile, A. y Oke, A. (2015). Assessment of facility management practices in public and private buildings in Akure and Ibadan cities, south-western Nigeria. Journal of Facilities Management, 13(4), 366-390. https://doi.org/10.1108/JFM-11-20140037

Organización Internacional de Normalización. (2015). Norma Internacional ISO 14001:2015. Sistema de Gestión Ambiental.

Organización Internacional de Normalización. (2018). Norma Internacional ISO 14067:2018. Gases de efecto invernadero - Huella de carbono de productos Requisitos y directrices para cuantificación. https://www.iso.org/obp/ui\#iso:std:iso:14067:ed-1:v1:es

Project Management Institute. (2017). Guía de los fundamentos para la dirección de proyectos. Guía del PMBOK (6. ${ }^{\mathrm{a}}$ ed.).

Secretaría Distrital de Ambiente. (2019). Programa Bogotá Construcción Sostenible. http://oab.ambientebogota.gov.co/es/ecourbanismo/programa-bogotaconstruccion-sostenible

Sridarran, P. y Gayani, N. (2016). Change management framework to enable sustainable outsourcing of facilities management services. Built Environment Project and Asset Management, 6(3), 317-331. https://doi.org/10.1108/BEPAM-08-2014-0041

Varajão, J., Colomo-Palacios, R. y Silva, H. (2017). ISO 21500:2012 and PMBOK 5 processes in information systems project management. Computer Standards \& Interfaces, 5o, 216-222. https://doi.org/10.1016/j.csi.2016.09.007

Yousefli, Z., Nasiri, F. y Moselhi, O. (2017). Healthcare facilities maintenance management: a literature review. Journal of Facilities Management, 15(4), 352375. https://doi.org/10.1108/JFM-10-2016-0040 